\title{
Réparer la base : l'avenir des soins primaires
}

$\mathrm{D}$ epuis longtemps, on considère les soins primaires comme l'assise de notre système de santé. Selon la définition classique de Barbara Starfield, il s'agit du «niveau d'un système de services de santé qui donne accès au système pour tous les besoins et les problèmes nouveaux, dispense au fil du temps des soins axés sur la personne (et non sur la maladie) et pour tous les problèmes sauf les plus rares ou inusités, et coordonne ou intègre les soins dispensés ailleurs ou par des tiers ${ }^{1} \gg$. Le paysage a toutefois changé sensiblement depuis quelques années. La baisse du nombre de diplômés de facultés de médecine qui choisissent la médecine familiale constitue la dimension la plus évidente de ce changement. Même si l'on a redoublé d'efforts pour renverser la tendance, cette année, $75 \%$ des postes non dotés après le premier cycle du jumelage canadien des résidents étaient en médecine familiale².

Si les soins primaires battent de l'aile, ce n'est pas faute d'efforts. Il y a des exemples tout à fait remarquables de médecins et de communautés qui ont créé de solides réseaux et pratiques de soins de santé primaires. Le Group Health Centre de Sault-Sainte-Marie, par exemple, fondé en 1963 et inspiré du modèle des anciens HMO américains (c.-à-d. coopératifs), répond maintenant aux besoins en soins primaires et en soins spécialisés de 56000 patients inscrits et a réalisé des progrès exemplaires dans la prise en charge des maladies chroniques ${ }^{3}$.

Il y a toutefois eu aussi des déceptions. En dépit du calibre de leurs services, les cliniques de soins primaires communautaires ambitieuses du Québec, les CLSC, qui s'étendent à la grandeur de la province, sont mal utilisées et n'ont pas instauré de changement structurel fondamental ${ }^{4}$. Il semble que l'on sous-estime toujours les obstacles à la prestation efficace des soins primaires, en dépit des recherches et des évaluations intensives des 30 dernières années. Les facteurs suivants, notamment, contribuent à la situation : programmes d'études en médecine et en sciences de la santé qui tendent de plus en plus vers la sur-spécialisation; accent mis sur la technologie et les données factuelles plutôt que sur les facteurs humains et la communication; grilles d'honoraires qui, négociées dans les coulisses de la politique médicale, favorisent les spécialistes plutôt que les généralistes; planification de la santé et élaboration de politiques qui accordent une faible attention aux problèmes régionaux et communautaires; et investissement insuffisant dans les systèmes d'information, si vitaux pour l'intégration et la coordination des soins primaires.

Ces obstacles sont fondamentalement sociaux et politiques. Les valeurs dominantes de la société sont évidentes dans notre recherche d'efficacité, dans l'importance que nous attachons à la formation des spécialistes et dans notre dépendance envers les partenariats publics-privés. Il est plus facile de présenter un argument en faveur d'un appareil de tomographie à émission de positrons qu'en faveur d'un organisme de soins primaires au cœur du centre-ville. Comment se fait-il toutefois que les gains évidents que pourrait produire une amélioration de l'informatisation en santé n'ont pas incité à investir adéquatement dans l'infrastructure du dossier de santé électronique? Un système de soins primaires efficace et viable repose sur la coordination et l'intégration des services et, par conséquent, sur le transfert efficient et fiable de l'information. Nous avons la technologie, mais pas tout à fait. Les dossiers médicaux sont accessibles électroniquement à peu de médecins (et à aucun patient), et la «carte à puce» du patient dépasse encore à peine le stade de l'idée intelligente. Imaginez ce qui se passerait si vous deviez consulter le directeur de la banque pour connaitre le solde de votre compte et si l'on vous disait qu'il faudra des jours, voire des semaines, pour consulter votre dossier. Un dossier de santé inaccessible devrait être tout aussi inimaginable de nos jours. Il est désolant de voir que nous n'avons pas réussi à financer adéquatement un cadre pancanadien pour le dossier de santé électronique afin de fournir des renseignements personnalisés, confidentiels et juste à temps aux patients et à leurs médecins.

L'érosion des soins primaires comme assise de notre système de santé et guichet d'accès à celui-ci aura pour effet que l'on continuera de mettre l'accent sur les soins secondaires et tertiaires coûteux et à forte consommation de technologie, et qu'il deviendra de plus en plus difficile de maintenir un système public universel de soins de santé. Du 16 au 19 mai, des experts et des responsables des politiques présenteront des recherches et des idées nouvelles au cours de la Conférence nationale sur les soins primaires à Winnipeg. Nous attendons avec impatience leurs propositions sur les rénovations et les réparations. - $7 A M C$

\section{Références}

1. Starfield B. Primary care: balancing health needs, services, and technology. $2^{\mathrm{e}}$ éd. New York et Oxford : Oxford University Press; 1998. p. 8-9.

2. Sullivan P. Students still ambivalent about family medicine. $7 A M C$ 2004;170 (9): 1380 .

3. Rachlis M. Prescription for excellence: how innovation is saving Canada's health care system. Toronto : HarperCollins; 2004. p. 101-5.

4. Romanow RJ. Guidé par nos valeurs : L'avenir des soins de santé au Canada. Saskatoon : Commission sur l'avenir des soins de santé au Canada; 2002. Disponible : www.commissionsoinsdesante.ca (consulté le 16 avril 2004). 